\title{
Anti-SIRT1 autoantibody is elevated in ankylosing spondylitis: a potential disease biomarker
}

\author{
Qiongyi Hu${ }^{1 \dagger}$, Yue Sun ${ }^{1 \dagger}$, Yuan $\mathrm{Li}^{2 \dagger}$, Hui Shi ${ }^{1}$, Jialin Teng ${ }^{1}$, Honglei Liu${ }^{1}$, Xiaobing Cheng ${ }^{1}$, Junna Ye ${ }^{1}$, Yutong Su${ }^{1}$, \\ Yufeng Yin ${ }^{1}$, Mengru Liu', Jiucun Wang ${ }^{2^{*}}$ and Chengde Yang ${ }^{1 *}$
}

\begin{abstract}
Background: Little is known about the presence of specific autoantibodies in ankylosing spondylitis (AS), an immunemediated inflammatory disease. The object of this study was to explore potential autoantibody profiles in AS patients.

Results: Levels of anti-SIRT1 autoantibodies were significantly higher in AS $(P<0.001)$ and psoriatic arthritis (PSA) $(P<0.01)$ patients but not rheumatoid arthritis $(R A)$ patients compared with healthy controls. Additionally, titers of anti-NAD-dependent protein deacetylase sirtuin-1(SIRT1) antibodies were significantly higher in AS patients than in RA $(P<0.05)$ and PSA $(P<0.05)$ patients. Moreover, levels of anti-SIRT1 $(P<0.001)$ antibodies were significantly higher during the first year in patients with hip joint involvement. The anti-SIRT1 antibody positivity rate was $18.9 \%$ in AS patients.

Conclusions: Our findings indicate that anti-SIRT1 autoantibodies may serve as a marker for diagnosing AS and predicting hip joint involvement at an early stage.
\end{abstract}

Keywords: AS, Anti-SIRT1 antibodies, Biomarker

\section{Background}

Ankylosing spondylitis (AS) is an immune-mediated, insidiously progressive form of seronegative spondyloarthritis (SpA) that is characterized by enthesitis. AS progressively leads to inflammation, bone erosion, new bone formation and ankylosis of sacroiliac, vertebral, and peripheral joints, with the ultimate radiographic appearance of a "bamboo spine" [1]. The pain and ankylosis that occur in this disease may cause considerable disability.

Although the etiology of AS is incompletely understood, familial and genetic factors are thought to be involved [2]. A surge of genome-wide association studies (GWASs) have been performed to identify associations

\footnotetext{
* Correspondence: jcwang@fudan.edu.com; yangchengde@sina.com ${ }^{\dagger}$ Qiongyi Hu, Yue Sun and Yuan Li contributed equally to this work. ${ }^{2}$ State Key Laboratory of Genetic Engineering and Ministry of Education (MOE) Key Laboratory of Contemporary Anthropology, Collaborative Innovation Center for Genetics and Development, School of Life Sciences, Fudan University, Shanghai 200438, China

1 Department of Rheumatology and Immunology, Ruijin Hospital, Shanghai Jiao Tong University School of Medicine, No. 197 Ruijin Second Road, Shanghai 200025, China
}

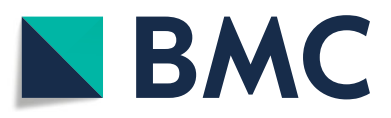

(c) The Author(s). 2018 Open Access This article is distributed under the terms of the Creative Commons Attribution 4.0 International License (http://creativecommons.org/licenses/by/4.0/), which permits unrestricted use, distribution, and reproduction in any medium, provided you give appropriate credit to the original author(s) and the source, provide a link to the Creative Commons license, and indicate if changes were made. The Creative Commons Public Domain Dedication waiver (http://creativecommons.org/publicdomain/zero/1.0/) applies to the data made available in this article, unless otherwise stated. the human leukocyte antigen allotype B27 (HLA-B27), the major risk factor for AS, as well as non-MHC genetics [2]. By delineating potential immunomodulatory pathways involving regulating innate and acquired immunity, such GWASs have provided considerable evidence for understanding the pathogenesis of AS [3]. The role of HLA-B27 in antigen presentation to T cells has been well established, and the association of $I L-7 R$, ZMIZ1, EOMES and RUNX3 variants with AS suggests the involvement of $\mathrm{CD}^{+} \mathrm{T}$ cell-mediated immunity [4]. Regardless, elucidating the roles of these factors in the immune system requires further study. Moreover, elevated numbers of Th17 cells and levels of IL-23 expression have been found in the peripheral blood of AS patients, indicating that $\mathrm{CD} 4^{+} \mathrm{T}$ cell-mediated inflammation contributes to the pathogenesis of AS. In addition to chronic inflammation, cartilage degeneration and new bone formation are key pathogenic features of AS. It has also been suggested that enhanced bone morphogenetic 
protein (BMP) and Wnt/ $\beta$-catenin signaling contributes to ankylosis and chondrogenesis in AS [5].

Despite sharing a susceptibility locus HLA genotype with other autoimmune diseases such as systemic lupus erythematosus (SLE) and Sjögren syndrome (SS) [6, 7], it remains controversial whether AS is an autoimmune disease with specific autoantibodies. Nonetheless, specific immune complexes have recently been found to be involved in AS pathogenesis [8], and levels of antibodies against connective, skeletal, and muscular tissue-related antigens [9], PPM1A [10], CD74 [11, 12], leukocytes [13], neutrophils [14], and some collagen proteins [15] are high in AS patients. Furthermore, an increased prevalence of anti-glycan antibody has been noted in AS and psoriatic arthritis (PsA) patients, with rheumatoid arthritis (RA) patients showing even higher prevalence [16]. In another study, AS patients were found to have higher levels of anti-flagellin antibody than a control group, suggesting an immune response to bacterial antigens in AS patients [17]. A comprehensive review on novel diagnostic autoantibodies in AS has been published, and targets of autoantibodies in AS include microbes, inflammatory factors and structural antigens [18]. Although these studies reveal a certain spectrum of autoantibodies in AS, the AS-related antibodies reported to date were based on small-study populations, and further validation is lacking. More evidence is needed to ascertain the autoreactivity associated with AS.

AS is considered an inflammatory rheumatoid disease, yet it differs from other autoimmune diseases with specific autoantibodies such as SLE and SS, and definitive evidence for autoantibodies in AS is lacking. The aim of this study was to explore potential autoantibody profiles in AS patients using a protein microarray expressing 19,349 recombinant human proteins. Autoantibodies targeting NAD-dependent protein deacetylase sirtuin-1 (SIRT1) in the protein microarray were then validated by an ELISA-based method, and significant differences in anti-SIRT1 antibody levels in AS patients with different clinical variables were assessed.

\section{Results}

\section{Global properties of observed antibodies in AS}

First, we explored global antibody profiles in serum from AS patients using a protein microarray displaying 19,349 immobilized recombinant human proteins. Analysis of the protein array data revealed 56 targets among the 2125 IgG antibodies expressed at levels 4-fold or greater in AS patients compared with healthy donors (Fig. 1a and Additional file 1: Table S1). Interestingly, functional analysis using the PANTHER pathway classification system indicated that the targets are mainly intracellular (Fig. 1b), similar to autoimmune diseases such as SLE. Additionally, the most significant enrichment in the antibody signature of AS patients was for terms of "catalytic activity" and "binding" (Fig. 1c). Further analysis of biological processes showed that most of these proteins are related to cellular, metabolic, stimulus response and developmental processes (Fig. 1d). Moreover, 13 proteins targeted by IgG antibodies were more than 10-fold higher in AS patients. The functions of 13 proteins were listed in Table 1. SIRT1 was the only antigen for which there was a significant highest level of IgG antibody in serum from AS patients compared to healthy controls. Interestingly, SIRT1 has been demonstrated to regulate bone metabolism; therefore, we concentrated on SIRT1 as a target.

\section{Distinct antibody expression levels in common rheumatic diseases}

To confirm the proteomic results, sera from 185 AS patients, 94 RA patients, 12 PsA patients, and 87 healthy controls were collected. The clinical characteristics of the subjects in each group are detailed in Table 2. As shown in Fig. 2, anti-SIRT1 antibody levels were significantly higher in AS patients than in healthy controls $(P<0.001)$. Among the disease controls, anti-SIRT1 antibody levels in AS patients were significantly different from those in RA $(P<0.05)$ and PsA $(P<0.05)$ patients $($ Fig. 2$)$. Moreover, sera from 35 patients with AS (18.9\%) and 18 with RA (19.1\%) were positive for IgG antibodies against SIRT1, whereas serum from only 1 patient with PsA (1\%) was positive for anti-SIRT1 antibodies.

\section{Anti-SIRT1 antibody levels were higher in female AS patients}

As sex hormones contribute to many autoimmune diseases including SLE, SS and AS [19], we compared levels of anti-SIRT1 antibodies between male and female AS patients. Remarkably, levels of IgG antibodies against SIRT1 were significantly higher in sera from female patients compared to male patients (Fig. 3, $P<0.05$ ). However, no gender difference for anti-SIRT1 antibody levels was observed among RA and PsA patients (data not shown). These results indicate that sex hormones may modulate the production and secretion of antibodies in AS patients.

\section{Elevated levels of anti-SIRT1 autoantibodies in AS patients with hip joint involvement at the early stage} We next analyzed statistical differences in serum antibody levels in AS patients with different disease variables. As shown in Fig. 4a, levels of anti-SIRT1 antibodies $(P<0.001)$ were significantly higher in patients with hip joint involvement during the first year, though anti-SIRT1 antibody levels were similar among AS patients regardless of disease duration (Fig. 4b). Furthermore, anti-SIRT1 levels were not significantly different 
between ESR-positive and -negative patients and did not correlate with ESR levels (Fig. 4c).

\section{Discussion}

In this study, we found serum levels of anti-SIRT1 antibodies to be significantly higher in sera from individuals with AS than from individuals with RA or PsA. In addition, levels of anti-SIRT1 antibodies were significantly higher in patients with hip joint involvement during the first year. The anti-SIRT1 antibody positivity rate among AS patients was $18.9 \%$, suggesting that anti-SIRT1 antibody is a potential novel biomarker in AS.

Although the pathogenesis of AS is not fully understood, it is commonly recognized that genetic susceptibility, environmental factors (especially the microbiome) and the immune system may be crucial factors in the development of this disease [20]. Indeed, multiple genetic variations have been found in genes encoding proteins essential for antigen presentation and macrophage-related phagocytosis, such as UBE2E3, ERAP1 and FCGR2A [1]. These data suggest that dysfunctional antigen processing and eradication occurs in AS. To date, a few studies have identified targets of autoantibodies in AS [9-15], though more definite evidence needs to be provided to verify the existence of autoreactivity in AS patients.

As imbalanced ossification is a hallmark in AS, we focused on proteins related to bone metabolism among 13 proteins targeted by IgG antibodies and showing more than 10-fold increase in AS patients. Interestingly, the anti-SIRT1 antibody level was highest in serum from AS patients. SIRT1, a widely distributed class III histone deacetylase, is involved in regulating $\mathrm{T}$ cell activation as well as tolerance and inflammation [21, 22]. Additionally, recent studies have indicated a potential role for SIRT1 in regulating bone metabolism [23], and reduced levels of SIRT1 in osteoblasts from patients with

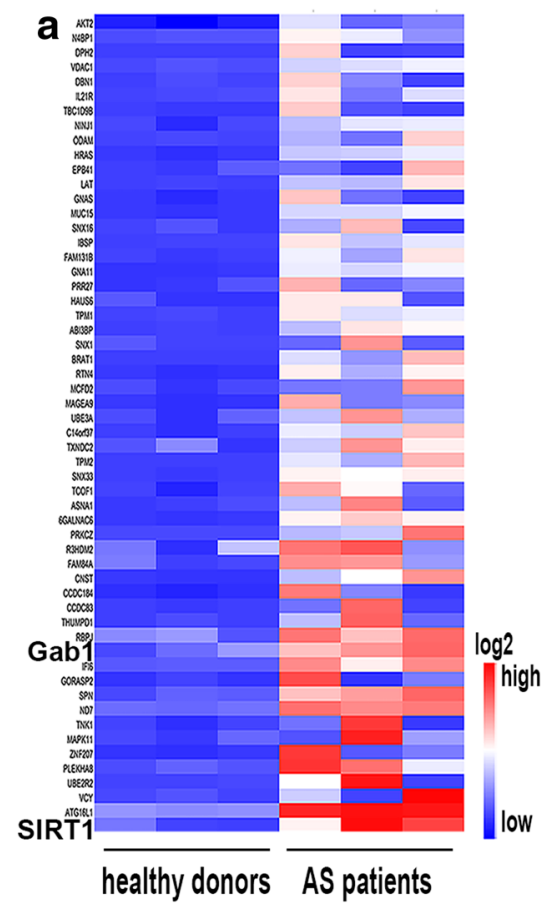

\section{b}

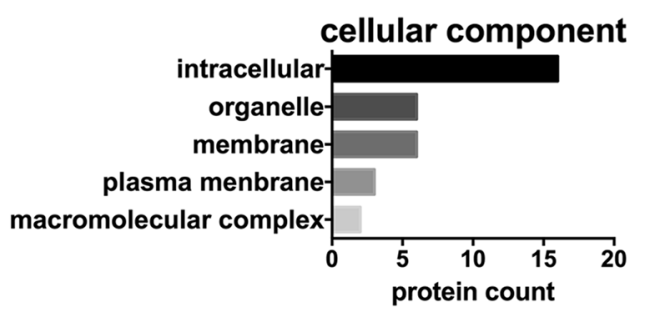

C

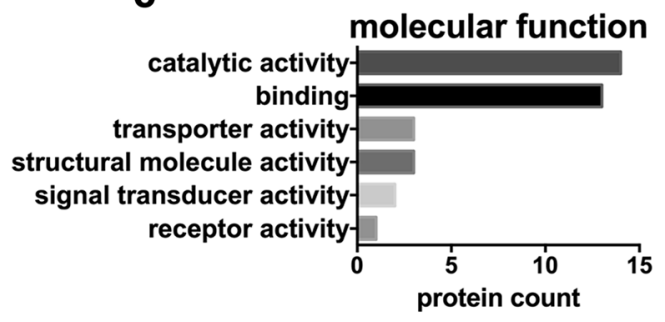

d

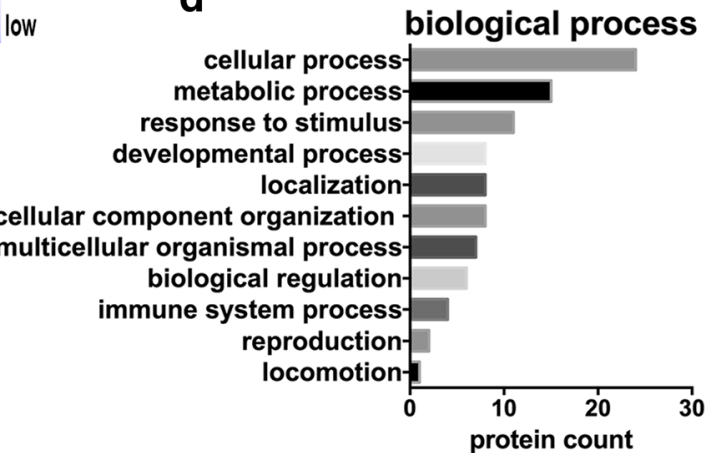

Fig. 1 Proteomic analysis of sera from AS patients. a. Heat map representing the 56 protein candidates targeted by lgG antibodies in AS patient sera with 4-fold or greater expression $(P<0.05)$ compared with healthy donors. The classification and clustering of different categories of cellular component (b), molecular function (c) and biological process (d) of all identified proteins were analyzed using PANTHER databases 
osteoarthritis, a disorder characterized by inappropriate osteogenesis of bone tissue, lead to increased expression of TGF- $\beta$ and sclerostin, which regulates bone mineralization via Wnt signaling [24]. Furthermore, MSCs isolated from SIRT1-deficient mice exhibit impaired differentiation into osteoblasts and chondrocytes [25], the major cells involved in the pathogenesis of AS. Moreover, SIRT1 is a negative controller of NF-kB, and well-demonstrated dysregulation of the NF-kB pathway, which is crucial for inflammation and the survival of osteoclasts and osteoblasts, has been reported in AS [26]. In our study, we found elevated levels of anti-SIRT1 antibodies in patients with hip joint involvement during the first year, indicating that SIRT1 may regulate erosive bone destruction in AS. We will explore the pathogenic role of SIRT1 in AS in the future.

PANTHER analysis revealed intracellular proteins to be the main targets of autoantibodies in AS. SIRT1 is mainly a cytoplasmic protein, and the autoreactivity against SIRT1 found in AS serum might reflect failed elimination of these antigens. More research will need to explain the role of these antigens in AS, and we regret the unavailability of spinal samples from AS patients to determine local expression of SIRT1 at affected joints.

Interestingly, our results showed that anti-SIRT1 levels were significantly higher in female patients compared to male patients. For some autoimmune diseases, sex hormones are among the most-studied contributory factors [27]. Unlike other autoimmune diseases with higher prevalence in females, such as SLE, SS and RA, AS is more prevalent in males than in females, and male patients also have more severe disease than do female patients [28]. It has been reported that testosterone might interact with SIRT1 to protect endothelial cells [29], though it remains to be determined how testosterone regulates SIRT1 and whether it might affect autoantibody production in AS.

Despite the presentation of SIRT1 as a self-antigen in AS, there were no significant differences in antibody levels when comparing diverse groups with low and high ESR or those with different disease durations. This finding indicates that the autoantibodies determined in the current study are irrelevant to inflammation in AS. Considering the functions of SIRT1 in bone formation as well as the positive correlation regarding autoreactivity, our study provides a basis for further research of the pathogenic mechanism of the immune system in AS.

AS has been recognized as a seronegative disease due to the lack of specific autoantibodies, which are the immunologic hallmarks of many autoimmune diseases such as SLE, SS and RA [30]. The present study reveals unique characteristics of AS compared with autoimmune diseases such as RA.

\section{Conclusions}

In summary, we found sera from AS patients to be extraordinarily distinct in terms of antibody reactivity toward antigens. In addition to the inflammatory response, autoantibodies produced by dysregulation of the immune system might constitute another feature of AS. Their core signature showed enrichment of a diverse array of proteins

Table 1 Targets of IgG antibodies with 10-fold or greater in AS patients

\begin{tabular}{lll}
\hline Name & Fold change & Functions \\
\hline SIRT1 & 29.3 & NAD-dependent protein deacetylase sirtuin-1 \\
ATG16L1 & 26.4 & Autophagy \\
VCY & 23.0 & Spermatogenesis \\
UBE2R2 & 20.5 & Ubiquitin-conjugating enzyme \\
PLEKHA8 & 19.9 & Cargo transport protein \\
ZNF207 & 16.8 & Kinetochore- and microtubule-binding protein \\
MAPK11 & 16.3 & MAP kinase signal transduction pathway \\
TNK1 & 14.9 & Negatively regulating RAS-MAPK pathway \\
ND7 & 14.7 & NADH dehydrogenase subunit 7 \\
SPN & 14.3 & Regulating multiple T-cell functions \\
GORASP2 & 13.1 & Golgi reassembly-stacking protein 2 \\
IFI6 & 11.6 & Regulating apoptosis, anti-viral activity \\
GAB1 & 11.1 & GRB2-associated-binding protein 1 \\
RBPJ & 11.0 & Playing a central role in Notch signaling \\
THUMPD1 & 10.6 & tRNA acetylation \\
CCDC83 & 10.2 & Coiled-coil domain-containing protein \\
CCDC184 & 10.1 & Coiled-coil domain-containing protein \\
CNST & 10.0 & Required for targeting of connexins to the plasma membrane \\
\hline
\end{tabular}


Table 2 Characteristics of the study cohort

\begin{tabular}{lllll}
\hline Variable & AS & RA & PsA & HC \\
\hline Number & 185 & 94 & 12 & $31-62(45.1)$ \\
Age (years) (median) & $15-70(34.6)$ & $14-54(41.1)$ & $10: 2$ & $20-50(35.2)$ \\
Gender ratio (male: female) & $139: 46$ & $12: 82$ & - & - \\
Disease duration (years) & $7.4(0.04-31)$ & - & - & - \\
Positive for HLA-B27 & $94.3 \%$ & - & 0 & - \\
Hips joint involvement (n, \%) & $57(30.8 \%)$ & 0 & $32.6 \pm 25.9$ & - \\
ESR (mm/h) (median) & $31.6 \pm 42.3$ & $31.2 \pm 25.7$ & & -
\end{tabular}

involved in bone metabolism, which may facilitate progression of AS, and included several novel antigens the function of which is not yet understood. These findings provide a framework for better definition of the role of the immune response and autoantibodies in the pathogenesis of AS. Moreover, we report the existence of anti-SIRT1 antibodies in sera from AS patients and the potential of anti-SIRT1 antibodies to serve as a disease biomarker for AS.

\section{Patients and methods}

\section{Subjects}

The first cohort of patients consisted of 10 treatmentnaïve AS patients who fulfilled the modified $1984 \mathrm{New}$ York criteria for AS [31]; 12 sex- and age-matched healthy donors were used as controls. Sera were collected and used for protein array analysis. The clinical characteristics of each group are summarized in Additional file 2: Table S2.

The second cohort of patients and controls consisted of 185 consecutive patients with AS, 94 patients with RA, 12 patients with PsA according to standard diagnostic criteria [32, 33]; 87 sex- and age-matched healthy donors were used as controls. Among the 185 AS

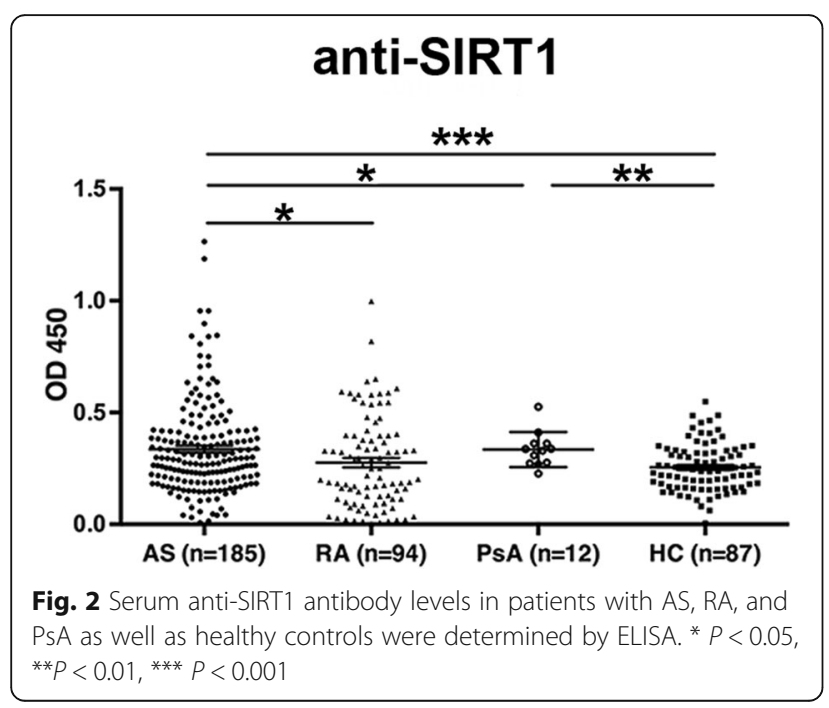

patients and 87 healthy controls, sera from 10 AS patients and 12 healthy controls were used for protein array analysis. Sera from the second cohort were used for ELISA analysis. All of the serum samples were collected at Ruijin Hospital between 2015 and 2016 and stored at $-80{ }^{\circ} \mathrm{C}$ until use. The study was performed in accordance with the Declaration of Helsinki and the principles of Good Clinical Practice. Biological samples were obtained under a protocol approved by the Institutional Research Ethics Committee of Ruijin Hospital (ID: 2016-62), Shanghai, China. All participants provided informed consent to participate in the study. Demographic and laboratory data were obtained by the Department of Immunology and Rheumatology.

\section{Serum antibody profiling using a human protein microarray}

The human proteome microarray (BC Biotechnology, USA) used in this study is composed of approximately 19,394 unique full-length recombinant proteins printed in duplicate. As a screening procedure, we employed protein array technology to detect new autoantibodies in AS using the first cohort of patients and healthy

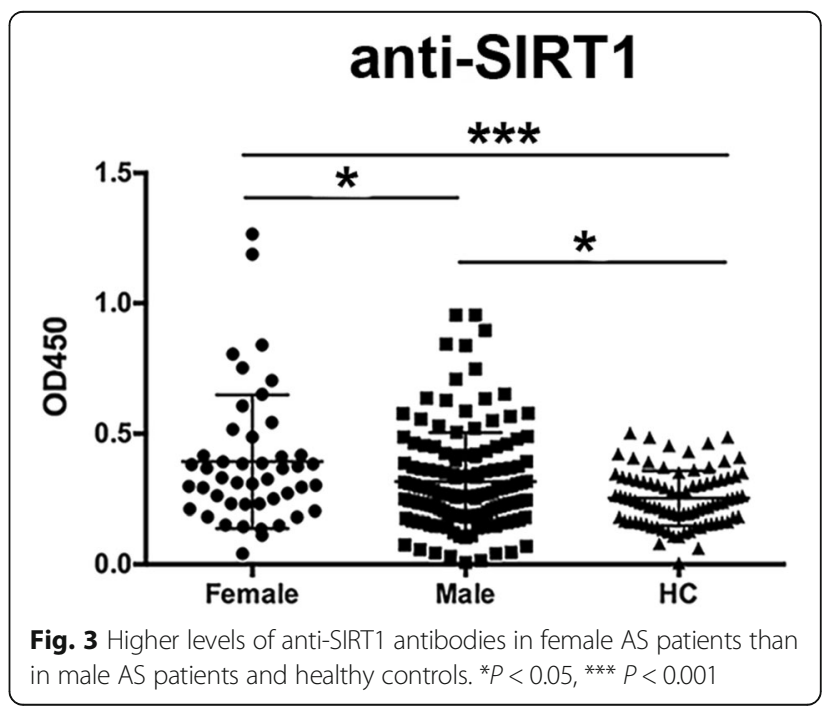



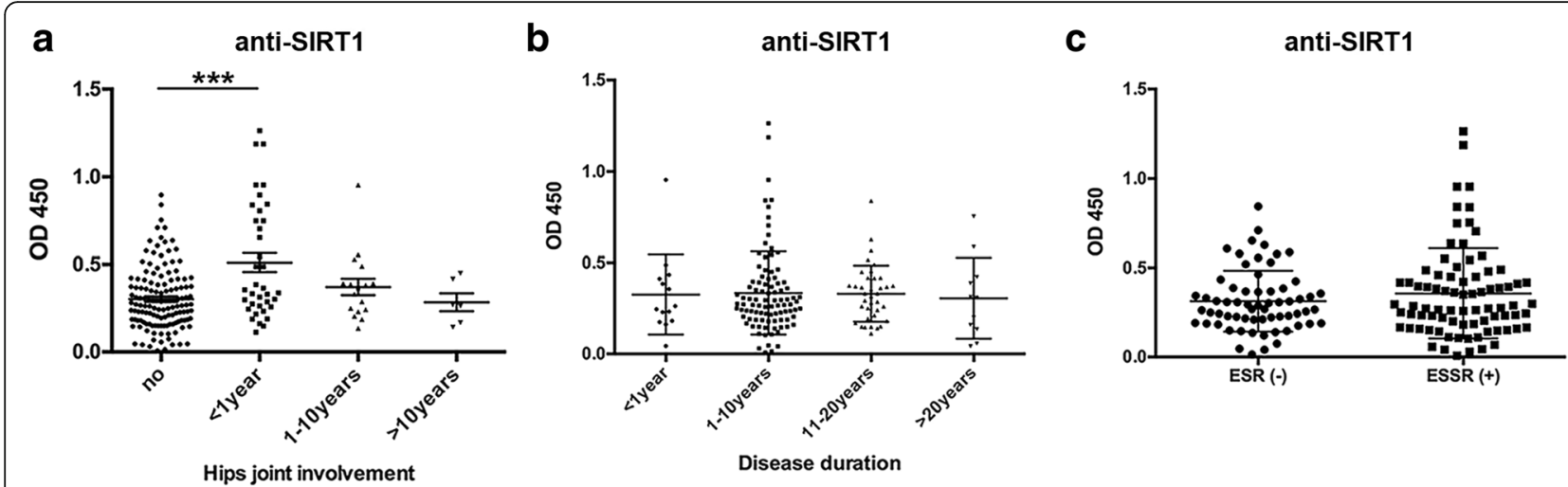

Fig. 4 Serum levels of anti-SIRT1 antibodies in AS patients with hip joint involvement, various durations of disease and ESR levels. a. Serum anti-SIRT1 antibodies in AS patients without hip joint involvement and with involvement duration of $<1$ year, 1-10 years and $>10$ years from symptom onset. b. Serum anti-SIRT1 antibody levels in patients with AS duration of $<1$ year, 1-10 years, 11-20 years and $>20$ years from symptom onset. c. No significant differences in serum anti-SIRT1 antibody levels in ESR-positive and ESR-negative groups were found. ${ }^{* *} P<0.001$

controls. Treatment-naïve patients with AS $(n=10)$ were divided into 3 groups, and sera of every group were mixed for detection. Sex- and age-matched sera from healthy donors $(n=12)$ divided into 3 groups were used as controls.

\section{Human protein microarray data analysis}

The protein microarray data analysis was conducted according to the procedure described by $\mathrm{Hu}$ et al. [34]. Median foreground and background intensities were obtained for each spot in the protein microarrays using Gene Pix Pro 6.0 Software. To execute background correction and normalize intra-array signal intensity, the raw intensity of each spot was defined as the ratio of the foreground to the background median intensity. To remove the negative effects of non-specific binding and spatial heterogeneity across the protein microarray, "normexp" [35], a background processing approach, was implemented in the $\mathrm{R} 2.15 .1$ package for background correction. The $P$ value was calculated for each protein using Fisher's test, and fold change was defined as the mean signal intensity ratio of AS patients to healthy donors.

\section{Identifying proteins differentially recognized by serum autoantibodies}

For each protein, spot signals were averaged across the groups. A statistical distribution of the signal intensities among the controls was calculated, from which the signal intensity mean and standard deviation were calculated. According to a previous autoantibody study by Meyer et al. [36], we identified the existence of 2125 proteins targeted by IgG antibodies by defining a cut-off of $\geq 2$ SD from the mean signal intensity of healthy donors and imposing a minimum prevalence of $2 / 3$ for the AS cases and a maximum prevalence of $1 / 3$ for the healthy donors. After filtering the 2215 protein hits with $P<0.05$ and fold changes of more than 4, 56 targets were identified as autoantigen candidates. Thus, for every target protein screened, the response of the control population formed a benchmark.

\section{Detection of serum autoantibodies}

Serum levels of anti-SIRT1 were measured by ELISA. In brief, 96-well plates were coated with $1 \mu \mathrm{g} / \mathrm{mL}$ SIRT1 (R\&D, Canada, USA) at $4{ }^{\circ} \mathrm{C}$ overnight. Nonspecific binding was blocked by incubating with PBS containing $5 \% \mathrm{BSA}$ at $37^{\circ} \mathrm{C}$ for $2 \mathrm{~h}$. The wells were then incubated with human serum $(1: 100)$ at $37{ }^{\circ} \mathrm{C}$ for $2 \mathrm{~h}$ and washed five times with PBS plus $0.05 \%$ Tween-20. Secondary horseradish peroxidase (HRP)-conjugated goat anti-human IgG monoclonal antibodies (Jackson ImmunoResearch Laboratories, Inc., West Grove, PA, USA) diluted to $1: 100,000$ were added to each well. After five washes with PBS plus $0.05 \%$ Tween $20,100 \mu \mathrm{L}$ of tetramethybenzidine substrate solution (Sigma-Aldrich) was added, and the samples were incubated at room temperature. The reaction was terminated by the addition of $50 \mu \mathrm{L}$ of $2 \mathrm{~N} \mathrm{H}_{2} \mathrm{SO}_{4}$ /well, and optical density (OD) was measured at $450 \mathrm{~nm}$. A cut-off of $\geq 2$ SDs of arbitrary units (AU) from the mean serum level of autoantibodies was used to qualitatively differentiate between positive and negative results.

\section{Statistical analyses}

All data were analyzed statistically using SPSS version 20.0 (SPSS Inc., Chicago, IL, USA). Quantitative data are expressed as the mean \pm SD. Differences between two groups were calculated using the Mann-Whitney $U$ test or unpaired Student's $t$-test, and differences between three or more groups were analyzed by one-way ANOVA and the Kruskal-Wallis test. Significant 
differences in antibody levels in AS patients with different clinical variables were evaluated using the MannWhitney $\mathrm{U}$ test or Kruskal-Wallis test. $P<0.05$ was considered significantly different.

\section{Additional files}

Additional file 1: Table S1. Targets of IgG antibodies in AS patients. (DOCX $16 \mathrm{~kb}$ )

Additional file 2: Table S2. Characteristics of AS patients and healthy donors in the protein microarray. (DOCX $14 \mathrm{~kb}$ )

\section{Abbreviations}

AS: Ankylosing spondylitis; BMP: Bone morphogenetic protein; GWASs: Genome-wide association; HLA-B27: Human leukocyte antigen allotype B27; PsA: Psoriasis arthritis; RA: Rheumatoid arthritis; SIRT1: NADdependent protein deacetylase sirtuin-1; SLE: Systemic lupus erythematosus; SpA: Seronegative spondyloarthritis; SS: Sjögren syndrome

\section{Acknowledgements}

Not applicable.

\section{Funding}

This work was supported by the National Natural Science Foundation of China (81671589, and 81601400), the Shanghai Sailing Program (16YF1407000), the National Basic Research Program (2014CB541801), and International S\&T Cooperation Program of China (2013DFA30870). The funding bodies had no role in the study design, data collection or analyses, decision to publish, or preparation of this manuscript.

\section{Availability of data and materials}

The datasets used and/or analyzed in the current study are available from the corresponding author upon reasonable request.

\section{Authors' contributions}

CY and JW designed the research strategy. QH and YS1 wrote the manuscript text and conducted ELISA. YL provided support for the bioinformatics analyses. HS and JT collected the clinical samples and data. $\mathrm{HL}$ and $\mathrm{XC}$ prepared Figs. 1, 2, 3, 4. JY and YS2 prepared the tables. YY and $\mathrm{ML}$ analyzed the data. All authors edited or commented on the manuscript. And all authors read and approved the final manuscript

\section{Ethics approval and consent to participate}

The study was performed in accordance with the Declaration of Helsinki and the principles of Good Clinical Practice. Biological samples were obtained under a protocol approved by the Institutional Research Ethics Committee of Ruijin Hospital (ID: 2016-62), Shanghai, China. All subjects provided written informed consent by themselves or by the parents on behalf of participants under the age of consenting for themselves.

\section{Consent for publication}

Not applicable.

\section{Competing interests}

The authors declare that they have no competing interests.

\section{Publisher's Note}

Springer Nature remains neutral with regard to jurisdictional claims in published maps and institutional affiliations.

Received: 1 March 2018 Accepted: 3 December 2018

Published online: 17 December 2018

\section{References}

1. Brown MA, Kenna T, Wordsworth BP. Genetics of ankylosing spondylitis-insights into pathogenesis. Nat Rev Rheumatol. 2016;12:81-91.
2. Evans DM, Spencer CC, Pointon JJ, Su Z, Harvey D, Kochan G, et al. Interaction between ERAP1 and HLA-B27 in ankylosing spondylitis implicates peptide handling in the mechanism for HLA-B27 in disease susceptibility. Nat Genet. 2011:43:761-7.

3. Smith JA, Colbert RA. Review: the interleukin-23/interleukin-17 axis in spondyloarthritis pathogenesis: Th17 and beyond. Arthritis Rheumatol. 2014;66:231-41.

4. Benjamin R, Parham P. Guilt by association: HLA-B27 and ankylosing spondylitis. Immunol Today. 1990;11:137-42.

5. Lories R. The balance of tissue repair and remodeling in chronic arthritis. Nat Rev Rheumatol. 2011:7:700-7.

6. Morris DL, Taylor KE, Fernando MM, Nititham J, Alarcon-Riquelme ME, Barcellos LF, et al. Unraveling multiple MHC gene associations with systemic lupus erythematosus: model choice indicates a role for HLA alleles and nonHLA genes in Europeans. Am J Hum Genet. 2012:91:778-93.

7. Fang K, Zhang K, Wang J. Network-assisted analysis of primary Sjogren's syndrome GWAS data in Han Chinese. Sci Rep. 2015:5:18855.

8. Tsui FW, Tsui HW, Las Heras F, Pritzker KP, Inman RD. Serum levels of novel noggin and sclerostin-immune complexes are elevated in ankylosing spondylitis. Ann Rheum Dis. 2014;73:1873-9.

9. Wright C, Sibani S, Trudgian D, Fischer R, Kessler B, LaBaer J, et al. Detection of multiple autoantibodies in patients with ankylosing spondylitis using nucleic acid programmable protein arrays. Mol Cell Proteomics. 2012:11:M9 00384

10. Kim YG, Sohn DH, Zhao X, Sokolove J, Lindstrom TM, Yoo B, et al. Role of protein phosphatase magnesium-dependent $1 \mathrm{~A}$ and anti-protein phosphatase magnesium-dependent 1A autoantibodies in ankylosing spondylitis. Arthritis Rheumatol. 2014;66:2793-803.

11. Baraliakos X, Baerlecken N, Witte T, Heldmann F, Braun J. High prevalence of anti-CD74 antibodies specific for the HLA class II-associated invariant chain peptide (CLIP) in patients with axial spondyloarthritis. Ann Rheum Dis 2014;73:1079-82.

12. Baerlecken NT, Nothdorft S, Stummvoll GH, Sieper J, Rudwaleit M, Reuter S, et al. Autoantibodies against CD74 in spondyloarthritis. Ann Rheum Dis. 2014;73:1211-4

13. Rosenberg JN, Johnson GD, Holborow EJ. Antinuclear antibodies in ankylosing spondylitis, psoriatic arthritis, and psoriasis. Ann Rheum Dis. 1979;38:526-8.

14. Locht H, Skogh T, Kihlstrom E. Anti-lactoferrin antibodies and other types of anti-neutrophil cytoplasmic antibodies (ANCA) in reactive arthritis and ankylosing spondylitis. Clin Exp Immunol. 1999;117:568-73.

15. Tani $Y$, Sato H, Hukuda S. Autoantibodies to collagens in Japanese patients with ankylosing spondylitis. Clin Exp Rheumatol. 1997;15:295-7.

16. Aloush V, Dotan I, Ablin JN, Elkayam O. Evaluating IBD-specific antiglycan antibodies in serum of patients with spondyloarthritis and rheumatoid arthritis: are they really specific? Clin Exp Rheumatol. 2018;25. [Epub ahead of print]

17. Wallis D, Asaduzzaman A, Weisman M, Haroon N, Anton A, McGovern D, et al. Elevated serum anti-flagellin antibodies implicate subclinical bowel inflammation in ankylosing spondylitis: an observational study. Arthritis Res Ther. 2013:15:R166.

18. Quaden DH, De Winter LM, Somers V. Detection of novel diagnostic antibodies in ankylosing spondylitis: an overview. Autoimmun Rev. 2016;15:820-32.

19. Fish EN. The X-files in immunity: sex-based differences predispose immune responses. Nat Rev Immunol. 2008:8:737-44.

20. Tam LS, Gu J, Yu D. Pathogenesis of ankylosing spondylitis. Nat Rev Rheumatol. 2010;6:399-405.

21. Kong S, Yeung P, Fang D. The class III histone deacetylase sirtuin 1 in immune suppression and its therapeutic potential in rheumatoid arthritis. Genet Genomics. 2013;40:347-54

22. Moon MH, Jeong JK, Lee YJ, Seol JW, Jackson CJ, Park SY. SIRT1, a class III histone deacetylase, regulates TNF-alpha-induced inflammation in human chondrocytes. Osteoarthr Cartil. 2013;21:470-80.

23. Cohen-Kfir E, Artsi H, Levin A, Abramowitz E, Bajayo A, Gurt I, et al. Sirt1 is a regulator of bone mass and a repressor of Sost encoding for sclerostin, a bone formation inhibitor. Endocrinology. 2011;152:4514-24.

24. Abed E, Couchourel D, Delalandre A, Duval N, Pelletier JP, Martel-Pelletier J, et al. Low sirtuin 1 levels in human osteoarthritis subchondral osteoblasts lead to abnormal sclerostin expression which decreases Wnt/beta-catenin activity. Bone. 2014:59:28-36.

25. Simic P, Zainabadi K, Bell E, Sykes DB, Saez B, Lotinun S, et al. SIRT1 regulates differentiation of mesenchymal stem cells by deacetylating betacatenin. EMBO Mol Med. 2013;5:430-40. 
26. Edwards JR, Perrien DS, Fleming N, Nyman JS, Ono K, Connelly L, et al. Silent information regulator (Sir)T1 inhibits NF-kappaB signaling to maintain normal skeletal remodeling. J Bone Miner Res. 2013;28:960-9.

27. Dragin N, Bismuth J, Cizeron-Clairac G, Biferi MG, Berthault C, Serraf A, et al. Estrogen-mediated downregulation of AIRE influences sexual dimorphism in autoimmune diseases. J Clin Invest. 2016;126:1525-37.

28. Taurog JD, Chhabra A, Colbert RA. Ankylosing spondylitis and axial Spondyloarthritis. N Engl J Med. 2016;375:1303.

29. Ota H, Akishita M, Akiyoshi T, Kahyo T, Setou M, Ogawa S, et al. Testosterone deficiency accelerates neuronal and vascular aging of SAMP8 mice: protective role of eNOS and SIRT1. PLoS One. 2012;7:e29598.

30. Ambarus C, Yeremenko N, Tak PP, Baeten D. Pathogenesis of spondyloarthritis: autoimmune or autoinflammatory? Curr Opin Rheumatol. 2012;24:351-8

31. Rudwaleit M, van der Heijde D, Landewe R, Listing J, Akkoc N, Brandt J, et al. The development of assessment of SpondyloArthritis international society classification criteria for axial spondyloarthritis (part II): validation and final selection. Ann Rheum Dis. 2009;68:777-83.

32. Aletaha D, Neogi T, Silman AJ, Funovits J, Felson DT, Bingham CO 3rd, et al. 2010 rheumatoid arthritis classification criteria: an American College of Rheumatology/European league against rheumatism collaborative initiative. Arthritis Rheum. 2010;62:2569-81.

33. Taylor W, Gladman D, Helliwell P, Marchesoni A, Mease P, Mielants H, et al. Classification criteria for psoriatic arthritis: development of new criteria from a large international study. Arthritis Rheum. 2006;54:2665-73.

34. Hu S, Xie Z, Onishi A, Yu X, Jiang L, Lin J, et al. Profiling the human proteinDNA interactome reveals ERK2 as a transcriptional repressor of interferon signaling. Cell. 2009;139:610-22.

35. Silver JD, Ritchie ME, Smyth GK. Microarray background correction: maximum likelihood estimation for the normal-exponential convolution. Biostatistics. 2009;10:352-63.

36. Meyer S, Woodward M, Hertel C, Vlaicu P, Haque Y, Karner J, et al. AlREdeficient patients harbor unique high-affinity disease-ameliorating autoantibodies. Cell. 2016;166:582-95.

Ready to submit your research? Choose BMC and benefit from:

- fast, convenient online submission

- thorough peer review by experienced researchers in your field

- rapid publication on acceptance

- support for research data, including large and complex data types

- gold Open Access which fosters wider collaboration and increased citations

- maximum visibility for your research: over $100 \mathrm{M}$ website views per year

At $\mathrm{BMC}$, research is always in progress.

Learn more biomedcentral.com/submissions 